FORMATION Formation emploi

Revue française de sciences sociales

111 | Juillet-Septembre 2010

Pêle-mêle

\title{
La première année en filière administration économique et sociale : motivations, abandons et attentes des étudiants
}

First year in an economic and social administration program: motivation, dropping out and student expectations

Das erste Jahr im Studiengang „Administration économique et sociale“

(Verwaltungswissenschaften): Motivation, Studienabbruch und Erwartungen

der Studenten

El primer año en la especialidad Administración económica y social :

motivaciones, abandonos y expectativas de los estudiantes

Alexandra Filhon

\section{(2) OpenEdition}

Journals

Édition électronique

URL : http://journals.openedition.org/formationemploi/3081

DOI : 10.4000/formationemploi.3081

ISSN : 2107-0946

Éditeur

La Documentation française

Édition imprimée

Date de publication : 15 septembre 2010

Pagination : 19-33

ISSN : 0759-6340

\section{Référence électronique}

Alexandra Filhon, «La première année en filière administration économique et sociale : motivations, abandons et attentes des étudiants », Formation emploi [En ligne], 111 | Juillet-Septembre 2010, mis en ligne le 10 octobre 2012, consulté le 30 octobre 2020. URL : http://journals.openedition.org/ formationemploi/3081; DOI : https://doi.org/10.4000/formationemploi.3081 


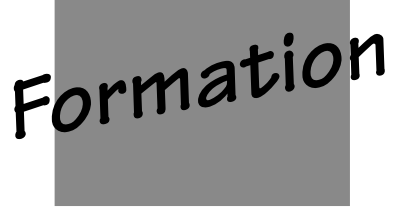

\title{
La première année en filière administration économique et sociale : motivations, abandons et attentes des étudiants ${ }^{1}$
}

\begin{abstract}
Alexandra Filhon *
La motivation à poursuivre jusqu'à la fin du premier cycle universitaire ne s'accompagne pas nécessairement d'un projet professionnel. Cependant, cette période d'indécision n'est pas nécessairement négative.
\end{abstract}

Les inégalités de formation post-bac résultent du type d'enseignement supérieur choisi, de la filière sélectionnée mais aussi de l'académie d'admission des bacheliers. Le système de formation supérieure est segmenté et l'université n'échappe pas à cette hiérarchisation des diplômes et des établissements (Felouzis, 2008). Ces choix de formation sont fortement liés au milieu social d'origine et au cursus antérieur. Ils s'inscrivent également dans un contexte politique marqué par le souhait du ministère de l'Éducation nationale de voir la moitié d'une classe d'âge accéder au niveau licence ${ }^{2}$. Toutefois, la façon dont les étudiants se projettent dans les études et leur réussite éventuelle en licence ne peuvent se comprendre sans saisir aussi leur rapport présent aux études.

\footnotetext{
1 Je remercie Céline Dumoulin pour ses relectures attentives de ce texte.

2 En 2007, Valérie Pécresse met en place le « Plan pour la réussite en licence » qui a comme principaux objectifs de limiter l'échec en première année universitaire et de permettre à $50 \%$ d'une génération d'obtenir un bac +3 .
}

La première année à l'université est décisive, les taux d'abandon y sont très élevés et certains jeunes ne réussiront pas à trouver leur "rythme », à s'adapter au système universitaire (Beaud, 1997). D'autres y parviendront mais ne seront pas satisfaits de la filière suivie. Ces rapports plus ou moins heureux à l'université et/ou à la formation envisagée pourront eux aussi influer sur leurs ambitions et leur perception de l'avenir. Dès lors, pourquoi les bacheliers s'orientent-ils vers

* Alexandra Filhon est sociologue, maître de conférences à l'université Paris Ouest Nanterre La Défense. Membre du Laboratoire Printemps (ProfessionsInstitutions-Temporalités)-CNRS et du CERPOS (Centre de recherche populations et sociétés), elle est aussi chercheure associée à l'Ined (Institut national d'études démographiques), dans I'unité "Identités et territoires ". Ses travaux portent principalement sur la genèse de l'université de masse et les pratiques linguistiques familiales. 


\section{Encadré 1}

\section{La filière AES (Administration économique et sociale)}

Créée en 1973, la filière AES symbolise la transformation de l'enseignement supérieur après 1968. Elle marque le début de la massification (Dumoulin, Filhon, 2008).

Elle est l'une des seules filières universitaires à combiner à parts à peu près égales trois pôles différents de connaissances : le droit, les sciences économiques et de gestion et les sciences humaines et sociales. Elle dispose également d'enseignements en informatique et en langues, deux domaines importants pour exercer des fonctions dans l'administration et la gestion.

Avec plus de 10000 entrants chaque année, cette filière regroupe la majorité de ses effectifs en licence. En effet, il s'agit à l'origine d'un parcours devant conduire à une insertion rapide sur le marché du travail. Les étudiants qui poursuivent leurs études après l'obtention de leur bac +3 s'inscrivent alors fréquemment en économie, droit, gestion ou encore en sociologie. C'est pourquoi, selon les universités, cette filière est rattachée à I'UFR (Unité de formation et de recherche) de Sciences sociales comme à I'université Versailles-Saint-Quentin en Yvelines (UVSQ) ou encore à I'UFR Droit, Économie, Gestion à Villetaneuse (Paris 13).

\section{Encadré 2}

\section{Trajectoires de réussite à l'université}

La recherche collective menée a pour objet l'analyse détaillée des trajectoires d'une cohorte d'étudiants inscrits en première année d'AES (Administration économique et sociale), en 2005, au sein de deux universités de la banlieue parisienne, Versailles-Saint-Quentin en Yvelines (UVSQ) et Villetaneuse (Paris 13). Ce suivi prévu sur cinq années permet de recueillir des données précises sur le cheminement des étudiants, sur les ressources qu'ils ont mobilisées pour poursuivre leurs études et sur les freins à leur réussite. Chaque année, la cohorte d'étudiants remplit un questionnaire et certains d'entre eux sont également interrogés par entretien. II s'agit de mieux comprendre comment ils gèrent leur nouveau statut d'étudiant, à quelles difficultés ils doivent faire face et de quelles ressources ils disposent. La démarche consiste à suivre les étudiants de leurs premiers pas à l'université jusqu'à leur insertion professionnelle. Trois thèmes sont plus particulièrement étudiés : les trajectoires scolaires et universitaires, les mobilités sociales et enfin les sociabilités (loisirs, relations amicales, engagements politiques, etc.).

Dans cet article, nous mobiliserons essentiellement les données du premier questionnaire, recueillies par voie postale auprès de 821 étudiants inscrits, en 2005/2006, en première année dans les deux universités. L'envoi de 1800 questionnaires a eu lieu en décembre 2005 ; ce qui signifie que les étudiants ayant déjà " décroché » à cette date ne font pas partie de la cohorte. Le taux de réponse est de plus de $45 \%$. Pour permettre la comparaison, les étudiants inscrits en droit et en économie ont aussi été enquêtés lors de cette première passation. La dimension comparative porte aussi sur l'inscription territoriale des étudiants. L'université de Versailles-Saint-Quentin en Yvelines (UVSQ) se situe au centre d'une communauté d'agglomérations où résident principalement des parents exerçant une profession intermédiaire ou cadre. À l'inverse, à l'université de Villetaneuse (Paris 13), le recrutement des étudiants est plus populaire.

$\left(^{*}\right)$ : Outre l'auteur, participent à cette recherche Céline Dumoulin, Sandrine Nicourd (responsable scientifique du projet), Olivia Samuel, Sylvie Vilter. Ce projet est financé par le conseil général du département 93 et l'OVE (Observatoire de la vie étudiante) de l'université Versailles-Saint-Quentin en Yvelines. Pour plus de précisions sur cette recherche collective, voir Dumoulin C., Filhon A., Nicourd S. (coord.) Samuel O. et Vilter S. (2009), Trajectoires d'études et d'insertion sociale des étudiants d'AES. Rapport intermédiaire pour l'université Paris XIII et le conseil général du département 93, 106 pages. 
l'université et choisissent-ils telle filière plutôt qu'une autre, notamment les étudiants inscrits en première année «Administration économique et sociale 》 (AES), filière qui marque le début de la massification de l'enseignement supérieur (encadré 1) ?

Les étudiants en AES sont particulièrement concernés par l'abandon de la formation au cours de la première année, puisqu'un sur deux ne poursuit pas au-delà. L'origine sociale et une plus grande distance avec la culture savante dispensée peuvent expliquer ce décrochage. Cependant, on ne peut limiter l'analyse à cette seule interprétation. Le rapport présent aux études, à savoir l'apprentissage du "métier d'étudiant », ainsi que la façon de se projeter dans son avenir professionnel peuvent également interférer. Alors que l'entrée sur le marché du travail devient une préoccupation grandissante pour ces jeunes adultes, le choix de s'orienter vers une filière pluridisciplinaire témoigne d'une certaine indétermination professionnelle. Cette indécision freine-t-elle leur projection dans les études?

Du passé structurant à la situation présente et future ${ }^{3}$, nous visons, à travers cet article, à révéler la pluralité des facteurs jouant sur les motivations et projections des jeunes adultes. Nous tentons de cerner ce qui peut favoriser ou au contraire limiter leur passage en deuxième année. En comparant les étudiants d'AES à ceux inscrits en droit et en économie, nous cherchons à saisir les spécificités de ces premiers afin de comprendre pourquoi ils abandonnent plus souvent leurs études que leurs camarades et pourquoi ils se projettent moins dans l'avenir. Notre propos est donc structuré en deux temps. Dans un premier temps, nous étudions les motivations des jeunes adultes à s'inscrire à l'université en AES. Dans un second temps, nous analysons les différences de projection des étudiants d'un cursus à un autre et d'une université à une autre.

Les analyses réalisées sont issues d'une recherche visant à suivre sur cinq années une cohorte entrée en première année d'études supérieures, en 2005, dans deux universités de banlieue parisienne (encadré 2).

\footnotetext{
${ }^{3}$ « L'action (la pratique, le comportement) est donc le point de rencontre des expériences passées individuelles qui ont été incorporées sous formes de schèmes d'action (schèmes sensori-moteurs, schèmes de perception, d'évaluation, d'appréciation, etc.) d'habitudes, de manières (de voir, de sentir et de faire) et d'une situation sociale présente » (Lahire, 1998).
}

\section{POURQUOI CHOISIR LA FILIÈRE AES ?}

Contrairement à certaines structures sélectives, l'université est un lieu qui se veut ouvert à tous. Cependant, un classement s'opère entre les différentes disciplines proposées $^{4}$. Nous étudions particulièrement ici la filière AES, et ce pour plusieurs raisons. Il s'agit tout d'abord d'une formation pluridisciplinaire et généraliste, présentée souvent comme une préparation aux concours de niveau licence, soit trois années post-bac. Créée en 1973 suite à plusieurs réformes universitaires, elle symbolise la transformation de l'enseignement supérieur après 1968. Elle marque le début de la massification de l'enseignement supérieur (Dumoulin, Filhon, 2008).

\section{Obtenir un emploi intéressant et un bagage de connaissances}

Globalement, en 2005/2006, dans les deux universités de banlieue parisienne étudiées, les étudiants, qu'ils soient inscrits en première année de droit, d'écogestion ou d'AES, attendent en premier lieu de leur formation qu'elle débouche sur un emploi intéressant (53\%); ensuite, qu'elle leur procure une bonne rémunération (41\%). Ce résultat est d'autant plus tangible pour les étudiants de droit et ceux issus de milieux favorisés (graphique 1).

Les étudiants en AES se distinguent des autres sur certains aspects. Ils attendent davantage que leurs camarades de droit ou d'éco-gestion une formation suffisamment générale pour les préparer à des concours (36\% sont dans ce cas, contre seulement $22 \%$ en eco-gestion et $26 \%$ en droit). Ils semblent également plus indécis. En effet, cette première année universitaire reste pour un quart d'entre eux une année de réflexion, soit deux fois plus que pour les étudiants inscrits en droit. Sans doute certains aspirent-ils avant tout à obtenir un emploi, sans autres prétentions c'est-à-dire sans trop formuler d'attentes concernant le secteur d'activités ou le salaire ; tandis qu'à

\footnotetext{
${ }^{4}$ « Le déclassement des diplômés de l'université, c'est d'abord le déclassement des formations les plus ouvertes et les plus démocratisées du point de vue de leur recrutement social »(Felouzis, 2008, p. 138).
} 


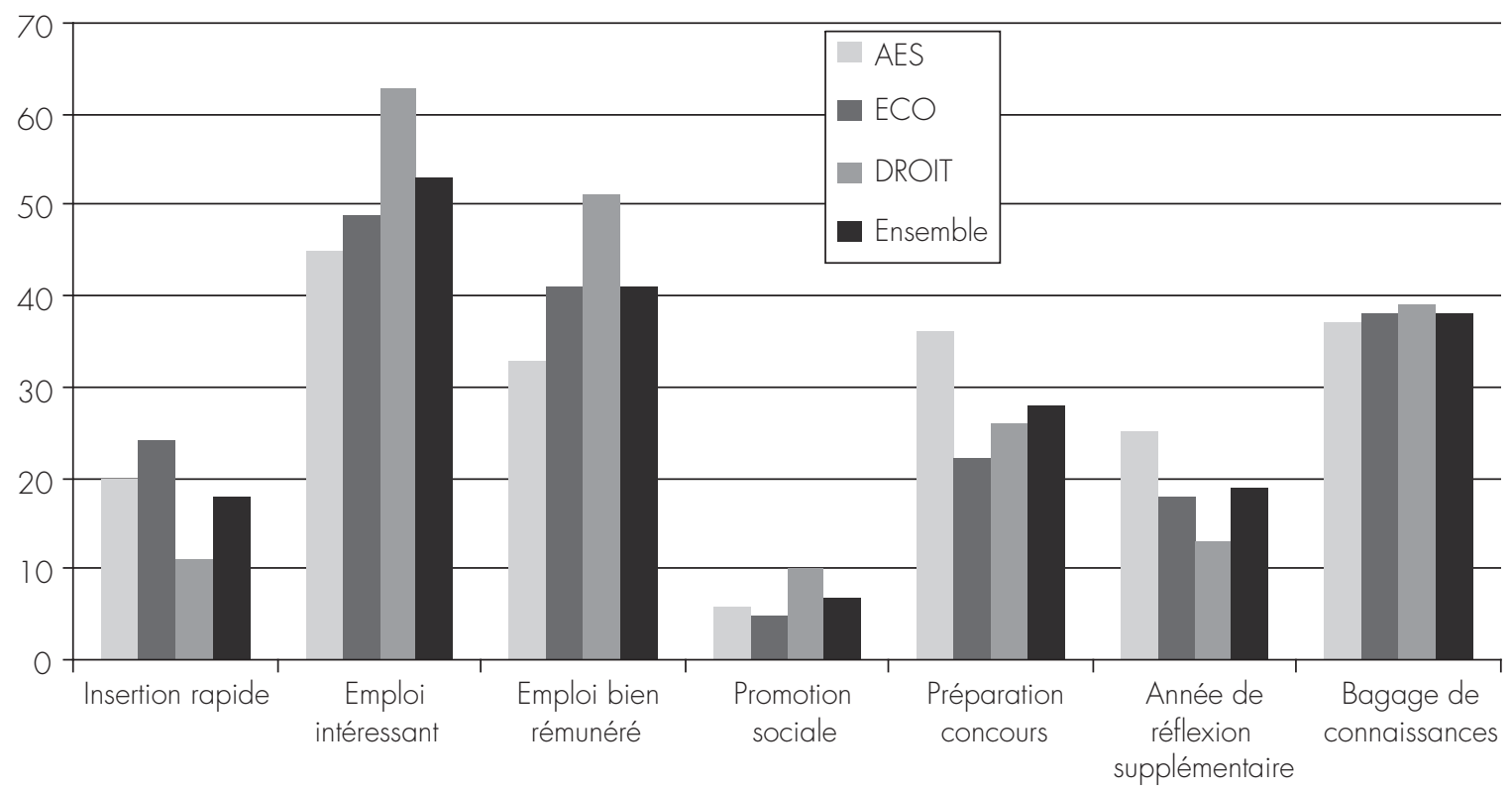

Source : Enquête «AES » 2006.

Note de lecture : À la question « qu'attendez-vous de votre formation actuelle ? », les étudiants - qui pouvaient indiquer deux réponses au maximum - souhaitent en moyenne en premier lieu un « emploi intéressant » et en second lieu un « emploi bien rémunéré ». Ils pouvaient également cocher les réponses : « une insertion rapide sur le marché du travail », " une promotion sociale », « une formation générale pour préparer des concours », « une année de réflexion supplémentaire pour choisir votre voie », « un bagage de connaissances », « autre ».

l'inverse, d'autres ne s'imaginent pas encore sur le marché du travail et se contentent de projets liés à leurs études. Pour les indécis, choisir une formation pluridisciplinaire permet alors de ne pas restreindre leurs perspectives professionnelles. Pour nombre d'étudiants, l'université demeure un lieu permettant d'acquérir de nouvelles connaissances. D'ailleurs, pour tous les étudiants, cette réponse arrive bien avant le souhait que la formation débouche sur une insertion rapide. Ainsi, les jeunes adultes qui s'inscrivent en première année à l'université semblent attendre de leur formation qu'elle leur apporte un savoir (37\%) bien plus qu'une place sur le marché du travail $(20 \%)$. En outre, les étudiants d'AES aspirent à ce que ce savoir s'inscrive dans le prolongement de leurs études secondaires, notamment à travers le maintien d'une pluralité de matières enseignées.

\section{Un choix « par défaut »?}

$\mathrm{Au}$ niveau national, parmi les bacheliers actuels, environ deux sur cinq se dirigent vers l'université.
Un an après leur entrée à l'université, plus de trois étudiants sur cinq poursuivent leurs études dans la même filière. Un étudiant sur dix s'est en revanche réorienté vers une autre formation de l'université et un sur quatre a quitté l'université. En AES, ce taux de sortie de l'université atteint $38 \%$, taux le plus élevé de toutes les filières universitaires. Seul un étudiant sur deux inscrit en AES en 2005 l'est encore l'année suivante $^{5}$. À l'inverse, en IUT (Institut universitaire de technologie), le taux de poursuite dans la même formation est le plus haut puisqu'il atteint $82 \%$ (DEPP, 2007) ${ }^{6}$.

\footnotetext{
5 Pour les deux cohortes suivies, des disparités importantes ont été relevées. À Paris 13, les taux d'abandon et de réorientations sont plus faibles puisque $42 \%$ des étudiants passent en deuxième année et $22 \%$ redoublent, tandis qu'à l'UVSQ, les taux s'élèvent respectivement à $38 \%$ et $6 \%$.

${ }^{6}$ Ces résultats, publiés dans Les étudiants-Repères et Références statistiques - Edition 2007, sont issus de l'enquête SISE (système d'information et de suivi des étudiants), Direction de l'Evaluation, de la Prospective et de la Performance (DEPP), Panel Bacheliers 2005.
} 
Nombre d'étudiants de première année d'AES espéraient accéder à une formation professionnalisante sélective mais n'ont pas été retenus ; ils ont donc choisi, dans un second temps, cette filière. Comme en IUT ou en STS (Sections de technicien supérieur), ce cursus pluridisciplinaire, qui s'inscrit dans le prolongement du secondaire, les intéresse particulièrement. Certains ne renoncent pas pour autant à ce souhait ; ainsi, environ un bachelier sur cinq, inscrit dans un premier temps en AES, se réoriente ensuite vers un IUT ou une autre filière universitaire. Renoncer à son parcours en AES ne signifie donc pas nécessairement abandonner ses études ; toutefois, les bacheliers technologiques rencontrent des difficultés pour accéder à ces formations professionnalisantes puisqu'ils ne constituent qu'un tiers de la population entrante (MEN, 2007). Nombre de jeunes adultes qui n'accèdent pas aux STS s'inscrivent en AES. Cette filière compte ainsi la plus grande proportion de bacheliers technologiques et professionnels. Dans les années 90 , ces bacheliers sortaient du système éducatif une fois ce diplôme obtenu; désormais, ils poursuivent au-delà car une insertion immédiate ne constitue plus la finalité de ces baccalauréats (Blöss et Erliche, 2000).

Malgré les possibles réorientations, la filière AES reste la formation universitaire qui enregistre le taux le plus élevé de sorties sans diplôme, certains étudiants renonçant définitivement à poursuivre des études supérieures. Près de quatre inscrits sur dix n'obtiennent pas de diplôme universitaire ni aucun autre diplôme (Lemaire, 2007), suivis en cela par les étudiants en sciences humaines ou en langues.

Dans l'ensemble des filières, environ $40 \%$ des étudiants obtiennent leur licence en trois ans (DEPP, 2006), réussissant sans retard leurs trois premières années d'études supérieures. En AES, la proportion est plus faible puisque environ un étudiant sur trois (35\%) obtient sa licence sans retard. Seuls les cursus en « Sciences fondamentales et applications » et en "Sciences économiques, gestion » enregistrent un taux de réussite inférieur. À l'inverse, arrivent en tête les « Sciences de la vie, de la santé, de la terre et de l'univers » et les « Lettres, sciences du langage, arts », disciplines dans lesquelles environ un étudiant sur deux obtient son diplôme en trois ans?

L'échec massif en première année, les réorientations sont liés en partie à une faible motivation d'étudiants qui sont là " faute de mieux ». Cependant, la réussite dépend sans doute aussi de la nature des savoirs dispensés d'une filière à une autre, des modalités d'organisation ou encore des choix pédagogiques adoptés selon les formations mais aussi selon les universités. Par exemple, on enregistre trois fois plus d'inscrits en première année d'AES à Villetaneuse qu'à Saint-Quentin en Yvelines. De même, la proportion de cours magistraux par rapport aux travaux dirigés diffère d'une université à l'autre : les cours magistraux sont en effet largement majoritaires à Paris 13 alors que la situation est inverse à l'UVSQ. L'autonomie croissante des universités explique cette grande variabilité d'organisation d'une université à une autre mais aussi d'une Unité de formation et de recherche (UFR) à l'autre. Ainsi, les taux d'encadrement, le tutorat ou encore les exigences aux examens varient parfois de façon significative entre les trois formations (AES, droit et éco-gestion), et ce sur le même site. Toutefois, comme l'ont déjà montré les travaux de Jarousse et Michaut (2001), il est complexe d'établir ensuite une corrélation entre ces disparités structurelles organisationnelles et pédagogiques et la réussite des étudiants. En effet, d'autres paramètres interviennent, comme par exemple le système de notation des enseignants et leurs propres attentes vis-à-vis du public étudiant. Ainsi, malgré des différences a priori notables entre les deux universités, les taux de passage de la première à la deuxième année sont quasiment identiques, soit environ deux étudiants sur cinq.

\section{Une orientation tardive dans la continuité des études antérieures}

Le choix de cette filière universitaire, sans grande conviction pour certains, peut avoir des conséquences sur la réussite en première année. Il rend compte également d'une moindre projection des jeunes dans les études et dans leur avenir professionnel.

${ }^{7}$ Les résultats publiés dans « Les étudiants », Repères et Références statistiques - Édition 2006 proviennent du Panel de bacheliers 2002 de la DEPP. 
Le moment où la décision de la formation post-bac a été prise est significatif ; il témoigne d'une plus ou moins grande anticipation qui peut en partie être liée à des projets. En effet, sans doute les jeunes adultes qui ont précocement fait le choix de leur devenir après l'année du bac avaient-ils une idée plus précise de leur parcours estudiantin, ce qui facilite leur projection. En ce sens, notre enquête de 2006 révèle que plus la décision de suivre un cursus en AES a été précoce, plus les étudiants envisagent des études longues. Parmi ceux qui se sont décidés le plus précocement, à savoir en seconde ou première, ils sont $59 \%$ à envisager poursuivre des études au-delà de la licence ; alors que la proportion est inférieure à $10 \%$, soit six fois moins, lorsque l'orientation post-bac a été décidée après l'obtention du diplôme.

Pour $60 \%$ des étudiants d'AES, le choix d'une inscription dans une formation plutôt que dans une autre est motivé par un projet professionnel, contre plus de $80 \%$ pour les étudiants de droit ou d'économie. D'ailleurs, la majorité de ces derniers déclarent que leur formation est en adéquation avec un éventuel projet professionnel ; quant aux jeunes inscrits en première année d'AES, ils estiment d'abord que leur formation concorde avec leurs études antérieures et leurs goûts personnels.

Ces réponses s'expliquent a priori par une moindre projection des étudiants d'AES dans l'avenir professionnel. Ils sont en effet nombreux à ne pas avoir d'idée, vague ou précise, de leur futur emploi : près de $40 \%$ contre seulement $27 \%$ des étudiants de droit. En revanche, les jeunes de première année d'AES ont a priori une idée plus précise de leur futur emploi que les étudiants en éco-gestion ( $25 \%$ contre $15 \%$ ). Mais ils sont loin derrière ceux inscrits en droit, dont plus de $38 \%$ ont, dès leur première année universitaire, une idée précise de leur future profession. L'éventail plus large des possibilités d'emploi de la filière AES explique en partie cette plus grande indécision ; il représente l'un des principaux motifs d'inscription dans ce parcours.

L'inscription dans cette filière pluridisciplinaire reflète donc l'échec de ces étudiants à intégrer un parcours sélectif, leur souhait d'acquérir un bagage de connaissances diversifié dans la continuité de leurs études dans l'enseignement secondaire et, enfin, une certaine indécision professionnelle. Ils espèrent en particulier pouvoir passer des concours administratifs à l'issue de leur licence, afin d'accéder à une certaine sécurité de l'emploi. Contrairement aux étudiants en droit, ils n'aspirent pas à une rémunération élevée. Ils souhaitent surtout ne pas devenir ouvrier et connaître des périodes de chômage.

\section{L'INDÉCISION PROFESSIONNELLE DES ÉTUDIANTS EN AES}

Dans un contexte généralisé de course aux diplômes, avec des taux de chômage élevés pour les jeunes sortis précocement du système scolaire, les étudiants envisagent de poursuivre leur cursus le plus longtemps possible. Dès leur première année universitaire, environ un tiers des étudiants espèrent poursuivre jusqu'à l'obtention d'un bac +5 . Seuls un quart des étudiants envisagent d'arrêter leurs études à bac +3 . Ils sont toutefois plus nombreux en AES.

\section{Les étudiants d'AES se projettent moins loin dans l'avenir}

Le choix de s'inscrire en AES n'est pas motivé par le souhait de s'insérer rapidement sur le marché $\mathrm{du}$ travail. Tout comme la poursuite d'études en sociologie, il s'agit davantage de formations dites « refuge », et de «passage à l'entrée dans les études supérieures » (Boyer et Coridian, 2002). Toutefois, contrairement à leurs camarades de droit et d'écogestion, ils envisagent d'arrêter plus tôt leur cursus : ils sont par exemple $40 \%$ en AES à se projeter au plus jusqu'au bac +3 , contre seulement $28 \%$ en éco-gestion et $26 \%$ en droit (tableau 1).

De même, environ un tiers des étudiants d'AES de première année ignorent jusqu'où ils poursuivront leurs études. Ils sont légèrement moins incertains en éco-gestion et d'autant moins en droit. Dans le cursus d'AES, les étudiants sont autant à envisager l'arrêt de leur cursus à bac +3 qu'au-delà. Et enfin, plus d'un sur dix prévoit déjà de ne pas achever sa licence d'AES. Les femmes semblent davantage envisager cette perspective et, globalement, elles se projettent moins loin dans l'avenir que leurs 
Tableau 1

Durée d'études envisagée selon la filière et le milieu social (\% en ligne)

\begin{tabular}{|c|c|c|c|c|c|}
\hline Filière & Avant bac +3 & À bac +3 & Après bac +3 & Ne sait pas & Ensemble \\
\hline AES & 13 & 27 & 27 & 33 & 100 \\
\hline dont issu d'un milieu défavorisé & 10 & 37 & 17 & 36 & 100 \\
\hline dont issu d'un milieu favorisé & 12 & 16 & 37 & 35 & 100 \\
\hline Eco-gestion & 10 & 18 & 42 & 30 & 100 \\
\hline dont issu d'un milieu défavorisé & 10 & 24 & 34 & 32 & 100 \\
\hline dont issu d'un milieu favorisé & 8 & 15 & 56 & 21 & 100 \\
\hline Droit & 9 & 17 & 46 & 28 & 100 \\
\hline dont issu d'un milieu défavorisé & 14 & 20 & 38 & 28 & 100 \\
\hline dont issu d'un milieu favorisé & 9 & 13 & 48 & 30 & 100 \\
\hline Ensemble & 11 & 21 & 37 & 31 & 100 \\
\hline
\end{tabular}

Source : Enquête « AES » 2006.

Champ : les totaux sont calculés à partir de la population étudiante totale alors que le « milieu favorisé » comprend uniquement les jeunes de père cadre, profession intermédiaire ou indépendant et que le « milieu défavorisé » inclut les bacheliers de père agriculteur, employé ou ouvrier.

homologues masculins. Selon le milieu social d'origine, des écarts significatifs apparaissent au sein de la même filière. En effet, les étudiants d'AES les plus défavorisés envisagent à $37 \%$ d'arrêter leurs études après l'obtention de leur licence, contre $16 \%$ seulement de leurs camarades mieux dotés socialement. Toutefois, les différences de projection persistent entre les formations. On constate ainsi que $37 \%$ des jeunes bacheliers inscrits dans la formation pluridisciplinaire et issus d'un milieu favorisé aspirent à poursuivre leurs études au-delà du bac +3 , contre respectivement $48 \%$ et $56 \%$ pour leurs homologues de droit et d'économie.

Des différences apparaissent entre les deux universités étudiées. Les étudiants formés à l'UVSQ envisagent de poursuivre leurs études plus longtemps que ceux inscrits à Paris $13(37 \%$ contre $29 \%$ ont l'ambition d'obtenir un bac +5 ). Ces différences reflètent principalement les écarts importants de milieu social d'origine d'un établissement à l'autre. L'effet de l'origine sociale apparaît donc clairement chez les étudiants de première année : les jeunes issus de milieux populaires se projettent moins loin dans l'avenir que ceux issus de milieux plus favorisés.

Les titulaires d'un bac professionnel ou technologique sont aussi ceux qui se projettent le moins loin dans leurs études. Ils semblent les plus incertains quant à leur devenir. Ils envisagent plus souvent de mettre un terme à leur parcours en AES avant la fin de la licence (tableau 2).

Les jeunes qui ont une idée de leur future profession estiment plus précisément la durée de leurs études, qu'il s'agisse a priori d'études courtes ou longues. Ces jeunes envisagent aussi le plus souvent d'interrompre leur cursus d'AES avant l'obtention de leur licence. Il s'agit, pour nombre d'entre eux, de souhaits de réorientation. Cela laisse penser que les jeunes adultes qui quittent la filière AES ne sont pas nécessairement les plus « désaffiliés ». À l'inverse, les étudiants d'AES n'ayant pas de projet professionnel sont nombreux à ne pas savoir jusqu'à quel niveau ils poursuivront leurs études. On retrouve donc l'incertitude du devenir professionnel dans la projection au niveau des études.

\section{Du passé structurant...}

Essayons à présent de mettre à jour les facteurs explicatifs de cette projection. Le milieu social d'origine joue un rôle décisif dans le choix de la structure scolaire, la filière envisagée, puis dans la perception des étudiants quant à leur avenir professionnel.

Dans l'ensemble, en France, trois étudiants sur dix inscrits à l'université ont un parent cadre, contre un sur dix seulement qui est enfant d'ouvrier, soit une représentation à peu près inverse de celle de la population 
Tableau 2

Durée d'études envisagée parmi les étudiants d'AES (Administration économique et sociale) selon la trajectoire scolaire antérieure, le sexe, le niveau d'études du père (\% en ligne)

\begin{tabular}{|c|c|c|c|c|c|c|}
\hline Sexe & Avant bac +3 & À bac +3 & Après bac +3 & Ne sait pas & Ensemble & Structure \\
\hline Homme & 10 & 29 & 30 & 30 & 100 & 30 \\
\hline Femme & 14 & 26 & 25 & 35 & 100 & 70 \\
\hline \multicolumn{7}{|l|}{ Bac obtenu } \\
\hline Bac général & 6 & 29 & 37 & 28 & 100 & 56 \\
\hline Bac techno & 20 & 25 & 14 & 40 & 100 & 34 \\
\hline Bac Pro & 26 & 26 & 6 & 42 & 100 & 10 \\
\hline \multicolumn{7}{|l|}{ Niveau d'études du père } \\
\hline$<$ au bac & 14 & 30 & 20 & 37 & 100 & 54 \\
\hline $\mathrm{Bac}$ & 10 & 35 & 30 & 25 & 100 & 7 \\
\hline $\mathrm{Bac}+1 \mathrm{a} \mathrm{Bac}+3$ & 14 & 14 & 49 & 24 & 100 & 12 \\
\hline $\mathrm{Bac}+4$ et + & 5 & 20 & 43 & 33 & 100 & 13 \\
\hline Ne sait pas & 18 & 33 & 18 & 33 & 100 & 13 \\
\hline \multicolumn{7}{|l|}{ Redoublements } \\
\hline oui & 16 & 25 & 25 & 34 & 100 & 56 \\
\hline non & 9 & 29 & 28 & 34 & 100 & 44 \\
\hline \multicolumn{7}{|c|}{ Prise de décision de suivre un cursus d'AES } \\
\hline Seconde ou première & 9 & 23 & 59 & 9 & 100 & 7 \\
\hline Terminale & 7 & 26 & 33 & 34 & 100 & 44 \\
\hline Après le bac & 27 & 24 & 9 & 40 & 100 & 27 \\
\hline $\begin{array}{l}\text { Lors d'une } 1^{\mathrm{re}} \text { ou } 2^{\mathrm{e}} \text { année } \\
\text { d'études après le bac }\end{array}$ & 8 & 31 & 29 & 31 & 100 & 17 \\
\hline Autre & 18 & 41 & 6 & 35 & 100 & 6 \\
\hline Ensemble & 13 & 27 & 27 & 33 & 100 & \\
\hline
\end{tabular}

Source : Enquête « AES» 2006.

Lecture : Parmi l'ensemble des étudiants d'AES interrogés, $70 \%$ sont des femmes. $25 \%$ d'entre elles aspirent à poursuivre leurs études au-delà du bac +3 contre $30 \%$ des hommes. De même, dans l'ensemble, $44 \%$ des inscrits en première année d'AES déclarent n'avoir jamais redoublé.

générale. À l'université de Saint Quentin, les étudiants sont davantage issus d'un milieu privilégié : environ quatre sur dix ont un père cadre ; à Paris 13, ils ne sont que deux sur dix. Ces écarts de milieu social sont visibles d'un établissement à un autre mais se creusent aussi selon les filières. Seul un étudiant sur dix inscrit en AES à Paris 13 déclare un père cadre contre un sur quatre à l'UVSQ.

De même, la moitié des pères d'étudiants inscrits en AES et interrogés dans l'enquête ont un diplôme inférieur au bac, avec des écarts significatifs entre les deux universités, et ce en défaveur des étudiants inscrits à Paris 13. Ces jeunes se projettent moins loin dans l'avenir que ceux dont les parents sont plus diplômés mais aussi que leurs camarades en droit ou en économie, issus du même milieu social. En effet, moins de $20 \%$ des jeunes en AES de père non bachelier envisagent des études au-delà du bac +3 , contre $40 \%$ pour leurs camarades en éco-gestion.

Plus souvent que les autres, les étudiants d'AES ont connu un passé scolaire jalonné d'échecs. En moyenne, plus de la moitié des jeunes adultes inscrits en AES ont redoublé avant leur entrée à l'université, soit davantage que dans les autres formations. La part des redoublants est plus importante à Paris 13 et parmi les jeunes diplômés d'un bac technologique ou 
professionnel. Toutefois, le redoublement ne constitue pas un facteur très discriminant dans la durée estimée des études, hormis pour ceux qui n'envisagent pas de poursuivre au moins jusqu'à l'obtention de leur licence (tableau 2).

L'AES représente donc une filière emblématique de la massification de l'enseignement supérieur. On y dénombre une forte proportion de jeunes issus de milieux populaires. Ils possèdent un bac technologique et ont parfois rencontré des difficultés au cours de leur cursus primaire et secondaire. Cette filière témoigne de la profonde transformation du paysage universitaire ces dernières années, qui accueille de nouvelles populations (Blöss et Erlich, 2000) issues de milieux sociaux plus défavorisés. La discrimination des publics selon l'origine sociale, qui se manifestait auparavant au niveau du collège, s'est étendue, dès les années 60 et 70, au lycée. Elle se fait ressentir plus tardivement au sein même de l'université. Progressivement, au fil $\mathrm{du} \mathrm{xx}^{\mathrm{e}}$ siècle, s'opère un déplacement des discriminations scolaires vers des niveaux d'études de plus en plus élevés (Merle, 1996). C'est pourquoi la distinction entre les étudiants ne s'opère pas seulement au regard de la durée de leurs études mais aussi de plus en plus en fonction de leur filière, qui constitue un critère de différenciation (Convert, 2003).

\section{... à l'incidence du rapport présent aux études}

Dans l'ensemble, les étudiants d'AES ne se sentent pas particulièrement «perdus » à l'université. En effet, huit sur dix ont répondu par la négative à cette question. Ils ne vivent pas ce passage du secondaire au supérieur comme une rupture (Montfort, 2003) ; ils perçoivent leur entrée à l'université comme une simple étape supplémentaire dans leur trajectoire scolaire. Pourtant, cette perception pose problèmes. En effet, pour certains, cette non-prise de conscience du changement important qui s'est opéré peut avoir des conséquences néfastes sur leur réussite universitaire (Beaud, 2002). Les étudiants d'origine populaire déclarent par exemple moins souvent se sentir perdus par rapport à leurs camarades de milieu plus favorisés. Cependant, ce ressenti ne semble pas avoir les mêmes conséquences selon les jeunes. En moyenne, près d'un tiers $(31 \%)$ des étudiants qui n'ont pas trouvé leur place à l'université ne souhaitent pas achever leur licence d'AES. Ils sont trois fois moins nombreux à vouloir abréger leur cursus lorsqu'ils se sont bien intégrés (tableau 3). Toutefois, cela concerne principalement les bacheliers les moins dotés socialement : lorsqu'ils se sentent perdus, les enfants de cadres inscrits en AES envisagent malgré tout de poursuivre leurs études jusqu'à l'obtention du master (pour $30 \%$ d'entre eux, contre $13 \%$ en milieu défavorisé).

Le déroulement de cette première année à l'université influe fortement sur la projection dans l'avenir des étudiants d'AES. Ceci est d'autant plus vrai pour les jeunes de parents ouvriers ou employés. Ils ne sont pas proportionnellement plus nombreux à déclarer des difficultés présentes ; toutefois, contrairement aux enfants de cadres, ce ressenti les conduit bien plus souvent à envisager de ne pas achever leur licence.

En AES, on trouve davantage d'étudiants faisant part de difficultés comparés à leurs camarades inscrits en droit ou en éco-gestion. Ce rapport différencié aux études ne peut s'analyser uniquement à partir de caractéristiques liées à l'étudiant. Il convient de prendre en considération l'incidence de la discipline choisie (Trinquier et Clanet, 2001). Comme nous l'avons dit, d'une formation à l'autre, l'organisation et la pédagogie peuvent fortement varier et la nature des savoirs peut également changer. La socialisation disciplinaire demandée n'est pas nécessairement la même, allant du bachotage à un rapport plus intellectualisé au savoir (Millet, 1999 ; Alava et Romainville, 2001).

Les étudiants qui envisagent de poursuivre leurs études le plus longtemps sont davantage satisfaits par leur formation. On peut supposer ici que l'intérêt pour la filière les conduit à envisager des études longues ; mais inversement, peut-être que l'implication dans les études des étudiants qui souhaitent se réorienter, voire qui ont déjà un projet professionnel à courte échéance, va être moindre. L'inscription universitaire n'est alors qu'un support, une étape en vue d'atteindre une autre structure d'études ou d'accéder rapidement au marché du travail. C'est pourquoi leur parcours universitaire est moins envisagé comme une source de connaissance en soi.

À titre de comparaison, Charles Soulié (2002), dans son étude menée auprès d'étudiants de sociologie, montre que le taux de satisfaction augmente lorsque 
ces derniers envisagent de poursuivre longtemps leurs études : $69 \%$ de satisfaction lorsqu'ils ne savent pas encore jusqu'où poursuivre, contre $75 \%$ pour ceux qui souhaitent obtenir un DEUG, $81 \%$ pour ceux qui envisagent une licence et enfin $92 \%$ pour la « minorité » se projetant au moins jusqu'en maîtrise.

Le rapport positif aux études ne s'accompagne pas nécessairement d'un projet professionnel. Dans sa thèse, Valérie Montfort (2003) explique que le terme de projet est souvent une notion peu pertinente pour comprendre le processus d'orientation des lycéens. Et comme nos résultats l'attestent aussi, une grande partie des bacheliers inscrits en AES n'a pas décidé d'aller à l'université à partir d'un projet professionnel précis. Leurs attentes vis-à-vis de la formation ne se réduisent donc pas à une insertion sur le marché du travail. Tout d'abord, nombre d'étudiants conçoivent l'université comme la continuité de l'enseignement secondaire. Ensuite, parmi les étudiants

Tableau 3

Durée d'études envisagée parmi les étudiants d'AES (Administration économique et sociale) en fonction du rapport présent aux études (\% en ligne)

\begin{tabular}{|c|c|c|c|c|c|c|}
\hline & Avant bac +3 & À bac +3 & Après bac +3 & Ne sait pas & Ensemble & Structure \\
\hline \multicolumn{7}{|c|}{ Cursus en AES en adéquation avec leurs goûts personnels } \\
\hline oui & 8 & 32 & 32 & 27 & 100 & 57 \\
\hline non & 19 & 21 & 17 & 44 & 100 & 43 \\
\hline \multicolumn{7}{|l|}{ Formation difficile? } \\
\hline Trop & 23 & 18 & 28 & 33 & 100 & 33 \\
\hline Pas assez & 10 & 24 & 57 & 10 & 100 & 9 \\
\hline Satisfaisante & 3 & 17 & 47 & 32 & 100 & 59 \\
\hline \multicolumn{7}{|l|}{ Formation abstraite? } \\
\hline Trop & 10 & 19 & 36 & 35 & 100 & 39 \\
\hline Pas assez & 24 & 18 & 44 & 15 & 100 & 14 \\
\hline Satisfaisante & 6 & 18 & 46 & 29 & 100 & 47 \\
\hline \multicolumn{7}{|c|}{ Étudiant d'AES se sent perdu à l'université ? } \\
\hline oui & 31 & 13 & 20 & 36 & 100 & 16 \\
\hline non & 10 & 29 & 27 & 34 & 100 & 84 \\
\hline \multicolumn{7}{|c|}{ Cursus en $\mathrm{AES}$ en lien avec un projet professionnel ? } \\
\hline oui & 5 & 37 & 36 & 23 & 100 & 58 \\
\hline non & 24 & 15 & 15 & 46 & 100 & 42 \\
\hline \multicolumn{7}{|c|}{ Les étudiants inscrits en AES ont-ils une idée de leur future profession? } \\
\hline Idée précise & 19 & 31 & 27 & 22 & 100 & 25 \\
\hline Idée imprécise & 12 & 30 & 25 & 33 & 100 & 35 \\
\hline Aucune idée & 10 & 22 & 27 & 42 & 100 & 39 \\
\hline \multicolumn{7}{|l|}{ Activité professionnelle } \\
\hline Aucune ou - de $2 \mathrm{~h} / \mathrm{sem}$. & 10 & 26 & 27 & 37 & 100 & 64 \\
\hline De 2 à $17 \mathrm{~h} / \mathrm{sem}$. & 12 & 32 & 30 & 26 & 100 & 27 \\
\hline Plus de 17h/sem. & 38 & 24 & 10 & 28 & 100 & 9 \\
\hline Ensemble & 13 & 27 & 27 & 33 & 100 & \\
\hline
\end{tabular}

Source : Enquête « AES» 2006.

Lecture : parmi l'ensemble des étudiants interrogés, $57 \%$ déclarent que leur cursus est en adéquation avec leurs goûts personnels. 32 \% d'entre eux aspirent à poursuivre leurs études au-delà du bac +3 , contre $17 \%$ de ceux dont le cursus n'est pas en adéquation avec leurs goûts personnels. 
inscrits en AES, la durée d'études envisagée diffère en fonction des attentes formulées vis-à-vis de la formation. Mais la causalité dans cette relation peut aussi être inverse. On peut donc tout à fait supposer que ce sont les attentes qui ont une incidence sur la projection dans les études. Logiquement, ce sont les étudiants incertains ou qui envisagent d'arrêter rapidement leur cursus d'AES qui déclarent plus souvent en moyenne que cette inscription en première année d'AES est avant tout une " année de réflexion ». Les jeunes adultes qui aspirent à une « insertion rapide» se projettent davantage jusqu'à la licence. Et enfin, les étudiants qui se projettent le plus loin font aussi directement référence à leur futur emploi, souhaitant qu'il soit intéressant et bien rémunéré.

Dans la typologie construite par Valérie Montfort (op. cit.) sur les étudiants en sciences et en AES, on retrouve ces trois types d'étudiants : d'abord, ceux pour lesquels l'université n'est qu'une passerelle vers une autre formation; ensuite, ceux pour qui ce parcours constitue la suite logique de leurs études et enfin, les jeunes qui ont un projet professionnel. $\mathrm{Si}$ les étudiants de première année en général mettent en avant leur choix d'aller à l'université comme une continuité du secondaire, les jeunes inscrits en AES se distinguent par leur plus grande indétermination. Ils ont choisi ce parcours pluridisciplinaire afin de ne pas se spécialiser. S'engager dans un cursus d'AES résulterait donc davantage d'un souhait d'accéder à un niveau de diplôme. Et en cela, ils valorisent moins le contenu même de leur formation. Sans doute que la diversité des matières enseignées leur laisse penser qu'ils conservent ainsi une large palette de perspectives professionnelles en attendant d'avoir une idée plus précise.

\section{Passé, présent, futur : les dimensions les plus discriminantes des trajectoires}

En adoptant un raisonnement « toutes choses égales par ailleurs », parmi l'ensemble des dimensions passées, présentes et futures des parcours de ces jeunes, on a cherché à faire émerger les dimensions les plus discriminantes. Il s'agit notamment de saisir si la projection dans l'avenir reste avant tout déterminée par l'origine sociale ou si elle s'explique également à travers le choix de formation.
Dans la probabilité de souhaiter ou non arrêter ses études avant l'obtention de la licence, plusieurs facteurs ont un effet propre (tableau 4). En premier lieu, les étudiants de l'UVSQ envisagent davantage cette option que ceux inscrits à l'université Paris 13. Pour comprendre cette distinction, il conviendrait sans doute d'examiner plus précisément l'offre de formations professionnalisantes et sélectives aux alentours de chacune des deux universités. On peut en effet émettre l'hypothèse que ces étudiants de première année de l'UVSQ envisagent prioritairement une réorientation.

Les quatre autres critères discriminants introduits sont : le type de baccalauréat, avoir ou non jugé la formation suivie « trop difficile», travailler plus de 17 heures par semaine ou pas, et enfin considérer que ses études sont en adéquation avec ses goûts personnels. Le type de baccalauréat obtenu a un effet très fort. Ainsi, les étudiants sortant d'un lycée technologique ou professionnel ont, « toutes choses égales par ailleurs ", six à sept fois plus de chances de ne pas envisager d'achever leur licence. Il semble également très important pour la poursuite du cursus que les enseignements suivis correspondent aux goûts du jeune adulte : la probabilité d'envisager de poursuivre au moins jusqu'au bac +3 est alors trois fois plus élevée. En revanche, cette projection jusqu'à l'obtention de la licence a minima n'est pas déterminée par le fait d'avoir déjà un projet professionnel. De même, on ne distingue pas davantage de différence significative entre les étudiants d'AES et ceux de droit ou d'éco-gestion. À situation comparable ( $c f$. tableau 4), la formation en AES n'engendre pas plus d'abandons ou de réorientations que les autres filières.

La trajectoire scolaire passée (le type de baccalauréat) conserve donc un poids considérable pour expliquer le maintien en études. Cependant, le second élément le plus décisif renvoie à la situation présente de l'étudiant. À partir d'un certain seuil, cumuler les études et l'emploi devient difficile. Le travail à mi-temps ou plus des étudiants résulte parfois d'un investissement pour l'avenir; envisageant une réorientation, au cours de cette année de transition, ils en profitent pour acquérir une expérience professionnelle ou épargner. Toutefois, d'autres travaillent par nécessité présente (payer leurs études, leur logement, etc.) et certains d'entre eux devront finalement arrêter leurs études pour entrer sur le marché du travail. 
Tableau 4

Poursuites d'études envisagées en première année universitaire

\begin{tabular}{|c|c|c|}
\hline \multicolumn{3}{|c|}{ Probabilités « toutes choses égales par ailleurs » de : } \\
\hline & $\begin{array}{c}\text { souhaiter arrêter ses études } \\
\text { avant le bac }+3\end{array}$ & $\begin{array}{c}\text { ne pas envisager de poursuivre } \\
\text { au-delà de bac }+3\end{array}$ \\
\hline & Odds ratio & Odds ratio \\
\hline \multicolumn{3}{|l|}{ Université } \\
\hline UVSQ & 2,0 & ns \\
\hline $\mathrm{P13}$ & ref & ref \\
\hline \multicolumn{3}{|l|}{ Filière } \\
\hline AES & ref & ref \\
\hline Eco-gestion & ns & 0,5 \\
\hline Droit & ns & 0,5 \\
\hline \multicolumn{3}{|l|}{ Redoublements } \\
\hline oui & ns & ns \\
\hline non & ref & ref \\
\hline \multicolumn{3}{|l|}{ Sexe } \\
\hline Homme & ns & ns \\
\hline Femme & ref & ref \\
\hline \multicolumn{3}{|l|}{ Bac obtenu } \\
\hline Bac général & ref & ref \\
\hline Bac techno & 6,3 & 1,9 \\
\hline Bac Pro & 7,1 & 4,6 \\
\hline \multicolumn{3}{|l|}{ Milieu social d'origine } \\
\hline Défavorisé & ns & 1,5 \\
\hline Favorisé & ref & ref \\
\hline \multicolumn{3}{|l|}{ Projet professionnel } \\
\hline oui & ns & 0,5 \\
\hline non & ref & ref \\
\hline \multicolumn{3}{|l|}{ Formation jugée « difficile » } \\
\hline oui & 1,7 & 1,7 \\
\hline non & ref & ref \\
\hline \multicolumn{3}{|l|}{ Bourse d'études } \\
\hline oui & ns & 1,5 \\
\hline non & ref & ref \\
\hline \multicolumn{3}{|l|}{ Travail } \\
\hline Aucune ou - de $2 \mathrm{~h} / \mathrm{sem}$. & ref & ref \\
\hline De 2 à $17 \mathrm{~h} / \mathrm{sem}$. & ns & ns \\
\hline Plus de 17h/sem. & 3,5 & 2,2 \\
\hline \multicolumn{3}{|c|}{ Études en adéquation avec goûts personnels } \\
\hline oui & 0,3 & 0,5 \\
\hline non & ref & ref \\
\hline
\end{tabular}

Source : Enquête « AES » 2006.

Lecture : Modèle logit. La situation de référence est signalée par « ref ». Les coefficients sont indiqués lorsqu'ils sont significatifs au seuil de

$10 \%$; au-delà, les résultats sont mentionnés comme étant non significatifs (ns).

L’odds ratio indique le rapport entre la chance de la modalité observée et celle de la situation de référence. Par exemple, « toutes choses égales par ailleurs ", les étudiants inscrits à l'UVSQ ont deux fois plus de chances de souhaiter arrêter leurs études avant l'obtention de leur licence. En revanche, à situation comparable, c'est-à-dire, à sexe, filière, redoublements, série de bac obtenu, milieu d'origine, présence d'un projet professionnel, sentiment que la formation est difficile et qu'elle est en adéquation avec ses goûts personnels, présence d'une bourse d'études et enfin à activité professionnelle équivalente, il n'apparaît aucune différence significative dans le second modèle entre les deux établissements. UVSQ : université Versailles Saint-Quentin. P13 : université Paris 13. 
Enfin, la troisième dimension la plus discriminante dans la poursuite d'études concerne les goûts personnels des jeunes. Ces goûts s'inscrivent bien sûr dans le prolongement de leur trajectoire passée mais ils témoignent également des ambitions de ces étudiants. Il s'agit de s'orienter vers une discipline non seulement parce qu'elle est susceptible d'offrir des débouchés professionnels mais aussi parce qu'elle correspond à des goûts personnels, des connaissances qu'ils souhaitent renforcer.

On observe enfin une évolution des facteurs explicatifs permettant de saisir une projection plus avant dans les études. Se fait jour une distinction entre les étudiants d'AES et les autres dans la projection $\mathrm{au}$-delà $\mathrm{du} b \mathrm{bc}+3$. Les étudiants en éco-gestion ou en droit ont une probabilité deux fois plus élevée que ceux inscrits en AES de souhaiter atteindre au moins le niveau bac +4 . On retrouve ici les mêmes éléments discriminants que précédemment, à quelques nuances près. Ainsi, par exemple, l'incidence du type de bac est moindre, en particulier pour les diplômés d'un bac technologique. Les titulaires d'un bac professionnel envisagent nettement plus souvent d'arrêter leurs études après la licence. Quant aux détenteurs d'un bac technologique, ils se rapprochent davantage des perspectives des diplômés de bacs généraux.

À ces premiers critères s'en ajoutent de nouveaux. Le projet professionnel n'a pas d'effet sur le fait de se projeter ou non jusqu'à bac +3 , mais il en a pour ceux espérant poursuivre au-delà. Ainsi, les étudiants se projetant le plus loin dans l'avenir sont aussi ceux qui ont, « toutes choses égales par ailleurs », une probabilité plus élevée d'avoir déclaré un projet professionnel. Assez logiquement, ce sont les jeunes qui ont une idée de leur future profession, qui se sont renseignés sur la durée nécessaire des études et donc savent déjà jusqu'où ils doivent poursuivre. En revanche, dans la projection plus ou moins ambitieuse qu'ils font en termes d'études, aucune différence significative n'est observée entre redoublants et non redoublants, une fois neutralisés les autres facteurs pouvant interférer ${ }^{8}$.

\footnotetext{
8 Dans une analyse similaire sur les étudiants de sociologie, Charles Soulié (2002) montre que ce " dynamisme des étudiants » décroît avec l'âge, caractéristique qui offre en fait un résumé de la trajectoire scolaire antérieure et révèle également des contraintes (mise en union, travail, etc.) qui interfèrent sur le bon déroulement des études universitaires. L'insatisfaction vis-à-vis de la formation rend ainsi compte de la « distance à la figure de l'étudiant canonique » (p. 27).
}

En définitive, parler de projet est sûrement abusif. En effet, nombre d'étudiants sont avant tout motivés pour poursuivre des études, même en l'absence de perspectives d'avenir très précises. C'est le cas notamment des jeunes adultes qui envisagent dans l'immédiat d'obtenir un bac +3 , et ce quelle que soit leur filière. Parmi les éléments de la scolarité passée, le type de baccalauréat obtenu est bien plus décisif en matière de projection dans l'avenir que le fait d'avoir redoublé. Plus largement, l'origine sociale interfère surtout entre ceux qui aspirent au moins à détenir un bac +4 et les autres. Par ailleurs, dans la projection en termes d'études, le rapport présent aux études (satisfaction vis-à-vis de la formation) doit lui aussi être pris en considération, indépendamment du milieu d'origine. Enfin, l'inscription dans la filière AES s'accompagne d'une projection vers des études « courtes ». L'une des finalités initiales de cette formation pluridisciplinaire, à savoir une insertion rapide sur le marché du travail, ne semble pas être la motivation première des jeunes qui ont choisi ce parcours. Toutefois, la seconde finalité, qui vise à ne pas trop spécialiser ces étudiants ni à les pousser vers des études en second cycle, semble y faire écho. Les étudiants inscrits en AES aspirent à obtenir un diplôme du supérieur qui soit suffisamment généraliste pour leur permettre de reporter leur projet professionnel. Pour autant, ils ne souhaitent pas poursuivre trop longtemps leur formation.

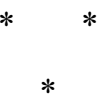

Les étudiants d'AES représentent pleinement les « nouveaux publics » (Soulié, 2002) de l'université. Celle-ci se distingue encore à ce jour des autres structures de l'enseignement supérieur. En effet, pour la plupart des filières, elle reste non sélective. Cette spécificité s'accompagne de la difficulté à gérer une population hétérogène, en particulier en première année. La filière AES, parce qu'elle est pluridisciplinaire et généraliste, attire un grand nombre d'étudiants aux profils variés. Certains sont là « faute de mieux » et les décrochages au cours de cette première année sont plus nombreux qu'au sein d'autres parcours. Le milieu social d'origine reste un facteur explicatif déterminant. Cependant, il ne peut suffire à appréhender ce rapport aux études supérieures. 
La motivation à poursuivre jusqu'à la fin du premier cycle ne s'accompagne pas nécessairement d'un projet professionnel. Certes, nombre d'étudiants sont dans une logique de bricolage au jour le jour de leurs carrières scolaires (Beaud, 2002) ; l'objectif étant, en particulier pour les jeunes de classes populaires, de prolonger un peu plus leurs études. Cependant, cette période d'indécision n'est pas nécessairement négative. Ce report d'un projet professionnel permet à certains de se tourner progressivement vers d'autres horizons que ceux qu'ils auraient sinon investis. Évidemment, la projection des étudiants dans l'avenir évolue au fil du cursus. Dans cette « période moratoire » (Galland, 1991) vécue par ces jeunes adultes, les fréquents bouleversements, les expériences inédites peuvent conduire à des réévaluations, à des renégociations de leur avenir et de leurs projections. Quitter le foyer parental, se mettre en couple ou à l'inverse rompre une union sont autant d'expériences qui peuvent influer sur leur trajectoire estudiantine. Il conviendrait donc d'explorer, à partir d'autres analyses de notre enquête de 2006 et des années suivantes, l'évolution des projets d'études et des projets professionnels des étudiants au regard de leur trajectoire familiale et personnelle.

Enfin, l'apprentissage du métier d'étudiant nécessite de parvenir non seulement à une affiliation institutionnelle mais aussi à une affiliation intellectuelle (Legendre, 2003). Pour ce faire, il importe de réussir à encadrer ces entrants. Il s'agit de favoriser leur apprentissage des « normes et techniques du travail intellectuel » (op. cit.) en engageant notamment une réflexion sur les pédagogies universitaires. L'une des difficultés réside dans la pluralité des modes d'organisation selon les établissements et les formations, pluralité qui s'est accrue depuis l'autonomie des universités. Au cours de ces quarante dernières années, l'enseignement supérieur s'est largement transformé, les profils étudiants se sont diversifiés. Pour tenter de faire face à ces évolutions, les réformes se sont multipliées, tout comme les stratégies pédagogiques. Toutefois, sans doute que les différents aménagements effectués de façon récurrente mériteraient d'être davantage évalués et réfléchis au niveau national.

\section{Bibliographie}

Alava S. et Romainville M. (2001), « Les pratiques d'études à l'Université », Revue française de pédagogie, $\mathrm{n}^{\circ} 136$, pp. 159-180.

Beaud S. (1997), « Un temps élastique. Étudiants des “cités" et examens universitaires », Terrain, $\mathrm{n}^{\circ} 29$, pp. 43-58.

Beaud S. (2002), 80\% au bac... et après ? Les enfants de la démocratisation scolaire, Paris, La Découverte.

Blöss T. et Erlich N. (2000), « Les nouveaux acteurs de la sélection universitaire. Les bacheliers technologiques en question ", Revue française de sociologie, $\mathrm{n}^{\circ} 41$.

Boyer R. et Coridian Ch. (2002), « Transmission des savoirs disciplinaires dans l'enseignement universitaire », Sociétés contemporaines, $\mathrm{n}^{\circ} 48$, pp. 41-61.
Convert B. (2003), « Des hiérarchies maintenues, espace des disciplines, morphologie de l'offre scolaire et choix d'orientation en France, $1987-$ 2001 », Actes de la recherche en sciences sociales, $\mathrm{n}^{\circ} 149$, septembre, pp. 61-73.

DEPP (2006), « Les étudiants », Repères et références Statistiques.

DEPP (2007), « Les étudiants », Repères et références Statistiques.

Dumoulin C. et Filhon A. (2008), « Construire l'université de masse. Genèse de la filière AES », Colloque "Ce que l'École fait aux individus », Nantes, 16 et 17 juin.

Dumoulin C., Filhon A., Nicourd S. (coord.) Samuel O. et Vilter S. (2009), Trajectoires d'études et 
d'insertion sociale des étudiants d'AES. Rapport intermédiaire pour l'université Paris XIII et le Conseil Général du département 93, 106 pages.

Felouzis G. (2008), « Des mondes incertains : les universités, les diplômés et l'emploi », Formation Emploi $\mathrm{n}^{\circ} 101$, pp. 135-147.

Galland O. (1991), Sociologie de la jeunesse. L'entrée dans la vie, Paris, Armand Colin, coll. «U », série « Sociologie».

Jarousse J.-P. et Michaut C. (2001), « Variété des modes d'organisation des premiers cycles et réussite universitaire », Revue française de Pédagogie, $\mathrm{n}^{\circ} 136$, pp. 41-51.

Lahire B. (1998), L'homme pluriel. Les ressorts de l'action, Paris, Nathan, Coll. « Essais et recherches ».

Legendre F. (2003), « Les étudiants fantômes. Les sorties précoces de l'université Paris 8 », Carrefours de l'éducation, juillet-décembre.

Lemaire S. (2007), « Les inscriptions à l'université : quel bilan ? » Note d'Information 07.10, Men-Direction de l'évaluation et de la prospective, octobre.
MEN (2007), « Les nouveaux inscrits en IUT à la rentrée 2005-2006 et la réussite en DUT » Note d'information, 07-18.

Merle P. (1996), « Les transformations sociodémographiques des filières de l'enseignement supérieur de 1985 à 1995. Essai d'interprétation », Population, Volume $51, \mathrm{n}{ }^{\circ} 6$, pp. 1181-1209.

Millet M. (1999), «Économie des savoirs et pratiques de lecture. L'analyse des formes du travail intellectuel étudiant en médecine et en sociologie », Éducation et Sociétés, $\mathrm{n}^{\circ} 4$, pp. 57-74.

Montfort V. (2003), Les étudiants de première année à l'université et le travail scolaire, l'exemple de deux filières : Sciences et AES, doctorat de sociologie, EHESS.

Soulié C. (2002), « L'adaptation aux nouveaux publics de l'enseignement supérieur : auto-analyse d'une pratique d'enseignement magistral en sociologie », Sociétés Contemporaines, $\mathrm{n}^{\circ} 48$.

Trinquier M.-P. et Clanet J. (2001), «Pratiques d'études et représentations de la formation chez les étudiants de première année : quelles limites à l'hétérogénéité ? », Revue française de Pédagogie, $\mathrm{n}^{\circ} 136, \mathrm{pp}$. 31-40.

\title{
Résumé
}

\section{La première année en filière administration économique et sociale : motivations, abandons et attentes des étudiants}

\author{
Alexandra Filhon
}

Cet article vise à étudier les profils et attentes des jeunes adultes qui choisissent de poursuivre leur cursus à l'université, en particulier ceux inscrits dans la filière "Administration économique et sociale » (AES). Pluridisciplinaire et généraliste, ce parcours attire des étudiants aux profils variés. Une analyse de leur trajectoire passée mais aussi de la situation présente et future révèle la pluralité des facteurs jouant sur leurs motivations et projections. En comparant les étudiants d'AES à ceux inscrits en droit et en économie, nous recherchons les spécificités de ces premiers afin de comprendre pourquoi ils abandonnent plus souvent leurs études et se projettent moins dans l'avenir. Ce cursus évite notamment à ces étudiants, indécis professionnellement, de se spécialiser et leur donne ainsi le sentiment d'avoir un large choix d'emplois.

Mots clés

Filière de formation, AES - Administration Économique et Sociale -, cheminement universitaire, étudiant Journal of Economic Literature: I 21, J 24 